\title{
Outcomes of retreatment with anti-PD-1 monotherapy after response to first course in patients with cutaneous melanoma
}

\author{
Eric D Whitman*,1, Emilie Scherrer², Wanmei Ou² \& Clemens Krepler² \\ ${ }^{1}$ Atlantic Melanoma Center, Atlantic Health System Cancer Care, 100 Madison Ave., Morristown, NJ 07960 USA \\ ${ }^{2}$ Merck \& Co., Inc., 2000 Galloping Hill Rd, Kenilworth, NJ 07033 USA \\ *Author for correspondence: Tel.: +1 973971 7111; Eric.Whitman@atlantichealth.org
}

\begin{abstract}
Aim: To determine outcomes of retreatment with anti-PD-1 monotherapy for melanoma. Materials \& methods: This retrospective study included adults with unresectable cutaneous melanoma who achieved stable disease (SD) or better after anti-PD-1 monotherapy and were retreated with anti-PD-1 monotherapy after $\geq 90$-day gap. We determined overall survival and real-world tumor response. Results: For 21 eligible patients, from retreatment initiation, median follow-up was 14.4 months (range, 2.6-34.5); median overall survival was 30.0 months $(95 \% \mathrm{Cl}$ : 14.4-not reached); 1-year survival was $100 \%$ (95\% Cl: $100-$ $100 \%)$; 2-year survival was $83 \%$ (48-96\%). Of 16 patients with recorded best real-world tumor response, ten $(63 \%)$ responded (complete/partial response); three achieved SD; three had progressive disease. Conclusion: Patients with advanced melanoma achieving SD/better after first-course anti-PD-1 monotherapy may benefit from retreatment.
\end{abstract}

First draft submitted: 7 April 2020; Accepted for publication: 28 April 2020; Published online: 15 May 2020

Keywords: immunotherapy $\bullet$ melanoma $\bullet$ outcomes research $\bullet$ real-world evidence $\bullet$ skin

The approvals in recent years of immune checkpoint inhibitors, as well as targeted BRAF/MEK inhibitors, have led to rapid changes in the treatment of advanced melanoma [1]. Ipilimumab was approved by the US FDA in 2011, followed by FDA approvals of pembrolizumab and nivolumab, monoclonal antibodies targeting programmed death 1 (anti-PD-1), in September and December 2014, respectively. Both pembrolizumab and nivolumab are currently approved in the USA for monotherapy of unresectable or metastatic melanoma, to be administered until disease progression or unacceptable toxicity.

In the era of chemotherapy, melanoma progression was common, particularly at more advanced stages $[2,3]$. As compared with prior systemic therapies for advanced melanoma, both pembrolizumab and nivolumab monotherapy improve tumor response rates and provide sustained disease control [4-7]. A recent report of 5 years' follow-up in the KEYNOTE-006 study found that 78\% of patients who had stable disease (SD) or better after a 2 -year course of pembrolizumab remained progression free 2 years later [5]. Durable responses have been achieved in oncology practice settings, outside of a clinical trial, as well $[8,9]$. Questions remain, however, about the optimal duration of anti-PD-1 monotherapy for advanced melanoma [5,10]. Moreover, if disease progression occurs after discontinuation of apparently successful anti-PD-1 monotherapy, an important clinical question is whether to initiate a second course of an anti-PD-1 agent. Will the patient's disease respond or at least be controlled with retreatment?

Preliminary evidence based on small patient numbers indicates that risk of disease progression is greater for patients who achieve a partial response (PR) versus complete response (CR) [8], and that patients with SD experience progression earlier than those who achieve PR or CR [5]. Further evidence suggests that retreatment with a second course of anti-PD-1 monotherapy can provide additional antitumor activity for patients with advanced melanoma who completed a first course with SD or better $[5,8]$. Our aim was to explore patient characteristics, treatment patterns and outcomes among patients with advanced cutaneous melanoma at US oncology practices who were retreated with second-course anti-PD-1 monotherapy after having responded or had controlled disease after a first course of anti-PD-1 monotherapy. 


\section{Materials \& methods}

\section{Data source}

This retrospective observational study was conducted using longitudinal data drawn from the Flatiron Health database in the USA [11]. Deidentified patient-level information in the database is collected via technology-enabled chart abstraction from electronic health records (EHRs) and includes structured data recorded electronically, such as laboratory test results and prescribed drugs, and unstructured data, such as freeform physicians' notes [12,13]. Prescribed lines of therapy are identified using oncologist-defined, rule-based extraction. At the time of this study, the database included information from over 280 oncology clinics and EHR data for analysis from more than 2.1 million patients. Data cutoff was 31 October 2018.

Approval for the Flatiron Health data abstraction protocol was obtained before study initiation from the Copernicus Group Institutional Review Board and included a waiver of informed consent for working with deidentified data. The data remained deidentified throughout the analyses to protect patient confidentiality. Flatiron Health, Inc., did not participate in these analyses.

\section{Patients}

Eligible patients were selected from the Flatiron Health advanced melanoma database, which requires at least two documented clinical visits on or after 1 January 2011, and a pathologically confirmed diagnosis of unresectable cutaneous melanoma at stage III or IV (International Classification of Diseases, Ninth Revision [ICD-9] code 172.x or ICD-10 diagnosis codes C43x or D03x) presenting on or after 1 January 2011, either at initial diagnosis or as locoregional or distant recurrence (metastatic recurrence) after an initial diagnosis of earlier stage disease. Patients in the advanced melanoma database who initiated anti-PD-1 monotherapy as pembrolizumab or nivolumab at age $\geq 18$ years on or after 4 September 2014 , and who were subsequently retreated with a second course of anti-PD-1 monotherapy (either pembrolizumab or nivolumab) were included in the study.

The second (retreatment) anti-PD-1 monotherapy course had to begin on or before 30 April 2018, to allow for a minimum of 6 months' potential follow-up. To identify two distinct anti-PD-1 monotherapy courses, a treatment gap of $\geq 90$ days between the last dose of the first course and the first dose of the retreatment course was required. In addition, to be eligible, patients had to respond to the first course of anti-PD-1 monotherapy with SD or better. Patients with progressive disease (PD) during the first course were excluded, as were patients who received another regimen in between the first and second anti-PD-1 courses. Patients with nonskin melanoma (ocular, subungal, mucosal, palmar and plantar) and those enrolled in a clinical trial at any time were also excluded.

\section{Study end points}

The main exposure of interest was the second (retreatment) course of anti-PD-1 monotherapy for advanced melanoma. The following outcomes with respect to the second course of anti-PD-1 monotherapy were determined: drug type (pembrolizumab or nivolumab), overall survival (OS), real-world time on treatment (rwToT) and real-world time to next treatment (rwTTNT). In addition, enhanced manual chart review was used to determine real-world progression (rwP) and tumor response (rwTR), and reasons for discontinuation of anti-PD-1 retreatment, described further below. We used the label 'rw' (real world) to denote study end points because treatment information, tumor response and progression were determined retrospectively from EHR data recorded in conjunction with clinical care.

We determined OS from the start of retreatment, using the Flatiron Health mortality 2.0 end point to identify the date of death $[14,15]$ and censoring patients with no recorded date of death at the last activity date.

Electronically registered medication orders and administrations were used to determine rwToT, defined as the length of time from first to last administration date of the second anti-PD-1 monotherapy course, as previously described [16]. Patients were considered to have discontinued at the last administration date of anti-PD-1 monotherapy retreatment if they had a record of initiating the next line of therapy, they died during the retreatment course or they had a $\geq 90$-day gap between the last anti-PD-1 monotherapy administration date and last known EHR-recorded activity date. Patients still on anti-PD-1 retreatment, meeting none of these discontinuation criteria, were censored at their last administration date. This end point is analogous to the 'time to treatment discontinuation' end point used by the Friends of Cancer Research pilot project for non-small-cell lung cancer (NSCLC), although the latter used a 120-day treatment gap instead of the 90-day gap used in the present study [17]. 
The rwTTNT end point was defined as the length of time from the first retreatment administration date to the date of initiating the subsequent treatment line or the date of death, and patients not receiving a subsequent treatment line or with confirmed death were censored at their last known activity date.

The rwP and rwTR end points were determined from a combination of structured and unstructured data, such as freeform text, using enhanced manual chart review. The rwTR end point was defined as the treating clinician's assessment of change in melanoma tumor burden based on radiologic evidence and occurring at least 30 days after initiation of retreatment. Assessments of change, captured using enhanced manual chart review, could be direct or indirect. Chart abstractors mapped each rwTR assessment as CR, PR, SD, PD, and other (pseudoprogression, indeterminate, not documented), defining $\mathrm{CR}$ as complete resolution of all visible disease, $\mathrm{PR}$ as partial reduction in size of visible disease without areas of increase, and $\mathrm{SD}$ as no visible change or a mixed response, as previously described in detail for a study of NSCLC using the Flatiron Health database [18].

Real world objective response rate (rwORR) was defined as the percentages of patients with CR or PR among those with documented tumor response information. For patients with multiple rwTR assessments during antiPD-1 retreatment, the best response was used for rwORR classification. The duration of response (rwDoR) was calculated as the length of time from the first documented evidence of CR or PR in the retreatment course until the first subsequent PD or death within the same course of treatment; patients without a rwP event or date of death within the retreatment course were censored at the last known rwTR assessment of CR, PR or SD.

The rwP end point, also captured using enhanced manual chart review, was defined as a distinct episode in which the treating clinician concluded that there had been growth or worsening of melanoma. The source evidence for rwP, assessed beginning at the date of metastatic diagnosis, included physicians' notes and radiologic and pathologic reports that described progression either directly or indirectly, as previously described for EHR-derived studies of NSCLC [18-20] The first date of observed progression was then used to determine real-world progression-free survival, defined as the time from initiation of the retreatment course to the first documented rwP event or death from any cause, whichever occurred first and excluding rwP events within 14 days after initiation of anti-PD-1 retreatment. Patients with no rwP or death were censored at their last recorded contact date, and those with no rwP starting a subsequent line of therapy were censored at initiation of the new treatment line [18].

For patients who discontinued the retreatment course of anti-PD-1 monotherapy, abstractors identified one or more reasons for anti-PD-1 discontinuation.

\section{Statistical analyses}

This was an observational study including only descriptive analyses; therefore, no power calculation was performed. Patient characteristics and outcomes were summarized using mean (standard deviation) or median (range) to describe continuous variables, as appropriate, and count and percentage to describe categorical variables, including for the rwTR categories of CR, PR, SD, PD and unknown.

The Kaplan-Meier method was used for all time-to-event analyses to estimate medians and associated 95\% CIs. In addition, restricted means at 1 year were calculated for rwToT, and at 1 and 2 years for rwTTNT, by calculating the area under the Kaplan-Meier curve from the starting time point to the time point of interest, as previously described [16].

Statistical analyses were performed using SAS 9.4 software (SAS Institute Inc., NC, USA).

\section{Results}

\section{Patients}

A total of 21 patients were identified in the advanced melanoma database as having initiated two sequential courses of anti-PD-1 monotherapy from 4 September 2014, through 30 April 2018, with at least 90 days between the last dose of the first course and the first dose of the retreatment course (Figure 1). Two patients received one course of each anti-PD-1 drug (pembrolizumab first and then nivolumab), while of the 19 patients who received the same drug in both courses, eight (42\%) received pembrolizumab and $11(58 \%)$ received nivolumab. Because of low patient numbers, we elected to combine for analysis the two subcohorts prescribed different and same anti-PD-1 drug during the two treatment courses.

At the time when patients initiated the retreatment course of anti-PD- 1 monotherapy, their median age was 74 years; 12 patients were male (57\%; Table 1). Of patients with available data, $1 / 18$ (6\%) had an elevated LDH level, and 4/14 (29\%) had ECOG performance status of 2 . Nine of 20 tumors (45\%) with known BRAF mutation status were $B R A F$-mutant; none of the tumors had been tested for PD ligand-1 expression (Table 1). 


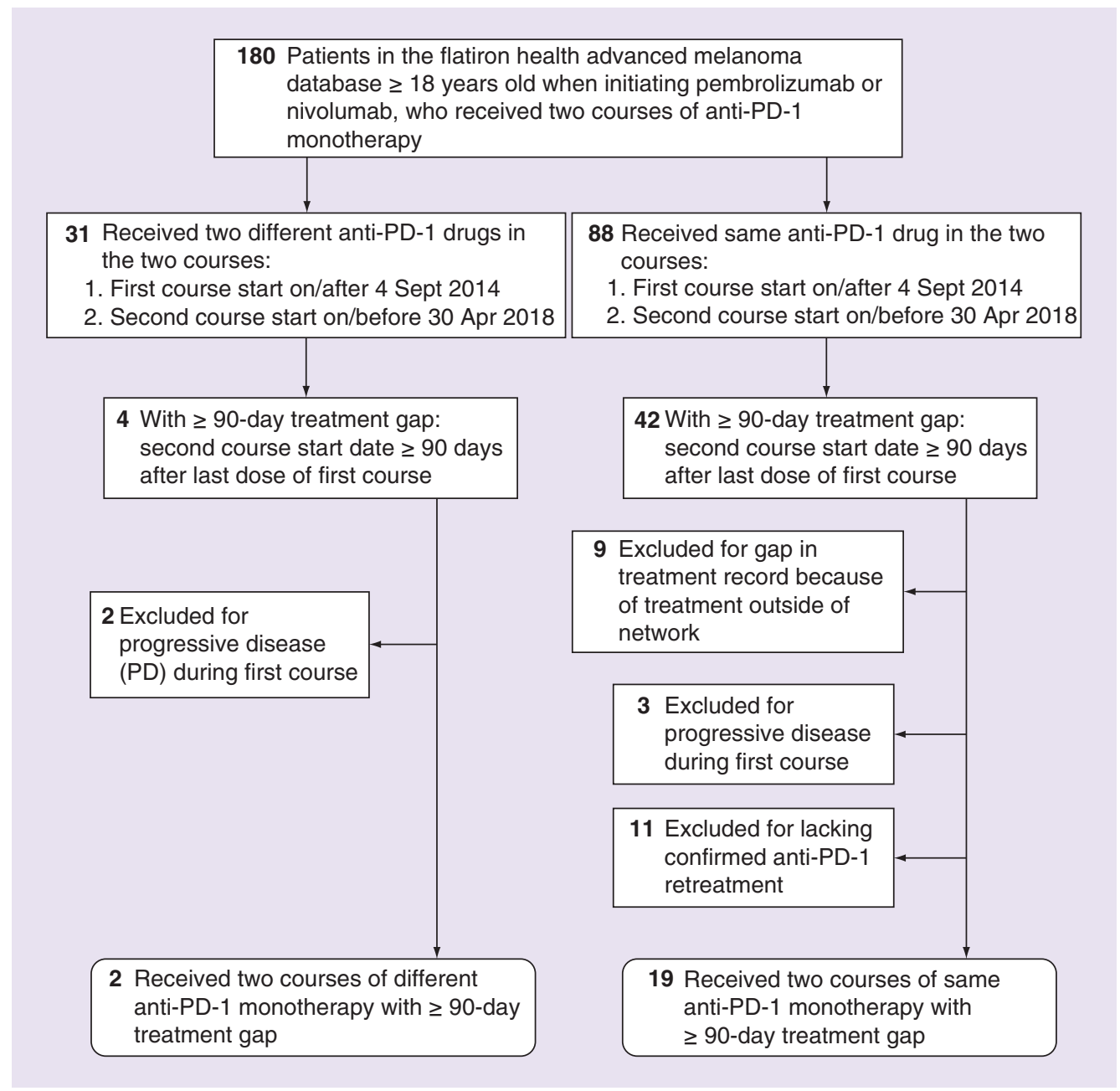

Figure 1. Selection of $\mathbf{2 1}$ eligible patients in the database. Patients included in the Flatiron Health advanced melanoma database (top box) had at least two documented clinical visits on or after 1 January 2011, and a pathologically confirmed diagnosis of unresectable cutaneous melanoma at stage III or IV presenting on or after 1 January 2011, either at initial diagnosis or as metastatic recurrence after an initial diagnosis of earlier stage disease.

Patients in the study were followed from initiation of the second course of anti-PD-1 monotherapy for a median of 14.4 months (range 2.6-34.5 months). Three of 21 patients (14\%) received the second course at academic medical centers, and the rest were retreated at community oncology clinics.

\section{Response to retreatment \& OS}

Median OS from the start of the retreatment course was 30.0 months (95\% CI: 14.4 not reached); estimated 1-year survival was $100 \%$, and 2-year survival was $83 \%$ (Table $2 \&$ Figure 2).

At data cutoff (31 October 2018), 10/16 patients (63\%) with known rwTR achieved best rwTR of CR or PR with retreatment (Figure $3 \&$ Table 3), and the median rwDoR for these patients was 6 months (Table 2). Three of 16 patients (19\%) patients had rwTR of SD, and three patients (19\%) had PD (rwTR was unknown for five patients). This equates to an $81 \%$ disease control rate (13/16 patients).

\section{Treatment history}

The first course of anti-PD-1 monotherapy was administered to 15 patients $(71 \%)$ as first-line therapy, to five patients $(24 \%)$ as second-line therapy, and to one patient (5\%) as third-line therapy (Table 4). Ipilimumab was the most common prior therapy, and two of three patients with $B R A F$-mutant melanoma who received prior therapy before initiating anti-PD-1 monotherapy had received a BRAF or BRAF/MEK inhibitor (Table 4). 
Table 1. Demographic and clinical characteristics of patients with advanced melanoma.

\begin{tabular}{|c|c|}
\hline Variable & $\mathbf{n}=\mathbf{2 1}$ \\
\hline Stage available at first diagnosis ${ }^{\dagger} \ddagger$ : & $17(81)$ \\
\hline - Stage II or lower & $10(59)$ \\
\hline - Stage III or IIIB & $3(18)$ \\
\hline - Stage IIIC & $2(12)$ \\
\hline - Stage IV & $2(12)$ \\
\hline Experienced metastatic recurrence ${ }^{\dagger}$ & $14(67)$ \\
\hline Time from initial to metastatic diagnosis, median (range), months ${ }^{\dagger}$ & $12.8(0-159)$ \\
\hline \multicolumn{2}{|l|}{ At initiation of retreatment with anti-PD-1 monotherapy } \\
\hline Male sex & $12(57)$ \\
\hline Age, median (range), years: & $74(34-84)$ \\
\hline$-<55$ & $5(24)$ \\
\hline$-55-74$ & $6(29)$ \\
\hline$-\geq 75$ & $10(48)$ \\
\hline \multicolumn{2}{|l|}{ Race data: } \\
\hline - White & $18(86)$ \\
\hline -Other & $3(14)$ \\
\hline US CB region, data available ${ }^{\ddagger}$ : & $18(86)$ \\
\hline - Northeast & $4(22)$ \\
\hline - Midwest & $4(22)$ \\
\hline - South & $6(33)$ \\
\hline - West & $4(22)$ \\
\hline Adjusted CCl score, mean (standard deviation) & $4.4(3.9)$ \\
\hline Median (range) & $6(0-12)$ \\
\hline \multicolumn{2}{|l|}{$B R A F$ mutation status: } \\
\hline - BRAF mutation positive & $9(43)$ \\
\hline - BRAF wild-type & $11(52)$ \\
\hline - Unknown & $1(5)$ \\
\hline ECOG PS data available ${ }^{\ddagger}$ : & $14(67)$ \\
\hline - ECOG PS 0-1 & $10(71)$ \\
\hline - ECOG PS 2 & $4(29)$ \\
\hline LDH level data available ${ }^{\ddagger}$ : & $18(86)$ \\
\hline - Elevated LDH level $\$$ & $1(6)$ \\
\hline History of brain metastasis & 0 \\
\hline Prescribed corticosteroids $\mathbb{I}$ & $5(24)$ \\
\hline Autoimmune condition" & $1(5)$ \\
\hline \multicolumn{2}{|c|}{ 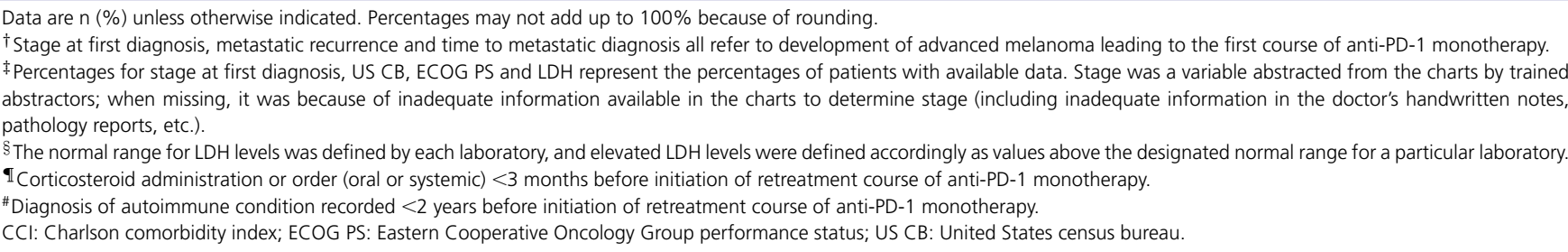 } \\
\hline
\end{tabular}

The median duration of the first course of anti-PD-1 monotherapy was 4.6 months (range, 0.7-24.4 months), and the median duration of the treatment gap was 5.3 months (range, 3.0-12.8 months; Table 4). The three most common reasons for the treatment gap between first and second anti-PD-1 monotherapy courses were adverse effect of therapy, other noncancer-related medical issue and drug holiday.

Median rwToT with the second course of anti-PD-1 monotherapy was 6.5 months, with a $21 \%$ on-retreatment rate at 1 year (Figure $2 \&$ Table 2). The median rwTTNT was 12.4 months, with nine patients continuing to a subsequent therapy (Table 4). 


\section{Table 2. Outcomes of retreatment with anti-PD-1 monotherapy}

\begin{tabular}{|c|c|}
\hline Variable & $\mathbf{n}=\mathbf{2 1}$ \\
\hline Patient follow-up from retreatment initiation, median (range), $\mathrm{mo}^{\dagger}$ & $14.4(2.6-34.5)$ \\
\hline \multicolumn{2}{|l|}{ Real-world time on (re)treatment (rwToT): } \\
\hline - rwToT, median $(95 \% \mathrm{Cl})$, months & $6.5(2.3-9.0)$ \\
\hline - Restricted mean rwToT at 1 year $(95 \% \mathrm{Cl})$, months & $6.1(5.1-7.1)$ \\
\hline -1-year on-treatment rate, $\%(95 \% \mathrm{Cl})$ & $21.4 \%(4.1-47.4 \%)$ \\
\hline \multicolumn{2}{|l|}{ Real-world time to next treatment (rwTTNT): } \\
\hline - rwTTNT, median $(95 \% \mathrm{Cl})$, mo & $12.4(3.6-\mathrm{NR})$ \\
\hline - Restricted mean rwTTNT at 1 year $(95 \% \mathrm{Cl})$, mo & $9.3(8.4-10.3)$ \\
\hline - 1-year rate of no next treatment & $61.6 \%(34.2-80.3 \%)$ \\
\hline-2 -year rate of no next treatment & $35.2 \%(11.8-60.0 \%)$ \\
\hline \multicolumn{2}{|l|}{ Duration of real-world tumor response $(r w D o R)^{\ddagger}$ : } \\
\hline - rwDOR, median $(95 \% \mathrm{Cl})$, mo & 6.0 (1.5-NR) \\
\hline - 1-year duration of response rate, $\%(95 \% \mathrm{Cl})$ & $34.3 \%(4.8-68.6 \%)$ \\
\hline \multicolumn{2}{|l|}{ rWPFS: } \\
\hline - rwPFS, median $(95 \% \mathrm{Cl})$, mo & $9.9(6.9-21.4)$ \\
\hline - 1-year rwPFS, \% (95\% Cl) & $41.2 \%(17.3-63.9 \%)$ \\
\hline - 2-year rwPFS, \% (95\% Cl) & $15.5 \%(1.2-45.6 \%)$ \\
\hline \multicolumn{2}{|l|}{ OS: } \\
\hline - OS, median $(95 \% \mathrm{Cl}), \mathrm{mo}$ & 30.0 (14.4-NR) \\
\hline - 1-year survival, \% (95\% Cl) & $100 \%(100-100 \%)$ \\
\hline - 2-year survival, \% (95\% Cl) & $83.3 \%(48.2-95.6 \%)$ \\
\hline
\end{tabular}

Table 3. Best real world tumor response to retreatment with anti-PD-1 monotherapy, stratified by response to first course of anti-PD-1 monotherapy $(n=21)$.

\begin{tabular}{|c|c|c|c|c|c|c|}
\hline \multirow[t]{2}{*}{ rwTR to 1st course } & \multicolumn{6}{|c|}{ rwTR to second course } \\
\hline & CR & PR & SD & PD & Unk & Total \\
\hline Complete response & 1 & 0 & 0 & 1 & 2 & 4 \\
\hline Partial response & 3 & 4 & 1 & 2 & 0 & 10 \\
\hline Stable disease & 0 & 2 & 2 & 0 & 3 & 7 \\
\hline Unknown & 0 & 0 & 0 & 0 & 0 & 0 \\
\hline Total & 4 & 6 & 3 & 3 & 5 & 21 \\
\hline
\end{tabular}

Data represent number of patients. For the patients with multiple rwTR assessments, the best response was used to classify the patient (CR $>$ PR $>$ SD $>$ PD). Patients with unknown rwTR had no evaluable assessment in the electronic health record.

CR: Complete response; PD: Progressive disease; PR: Partial response; rwTR: Real-world tumor response; SD: Stable disease; Unk: Unknown.

As detailed in Table 4, of the 11 patients with recorded reason for discontinuation of second-course anti-PD-1 monotherapy, five discontinued because of adverse effects of therapy, three because of disease progression and three because of disease-related symptoms not related to therapy.

\section{Discussion}

In this retrospective study, we found that 21 patients with advanced cutaneous melanoma who had SD or better after a first treatment course of pembrolizumab or nivolumab benefited from a second course of anti-PD-1 monotherapy. Overall, the survival rate was $100 \%$ at 1 year, and median OS was 30 months. For the 16 patients evaluable for rwTR, the response rate to retreatment was $63 \%(C R+P R)$, and disease control $(C R+P R+S D)$ was recorded for $81 \%$. Of note, to be included in the study, these patients needed to be eligible to receive anti-PD-1 monotherapy (with regard to performance status, etc.), as well as to live long enough to receive a second line of therapy after a minimum of 90 days' gap in therapy after the first line. 

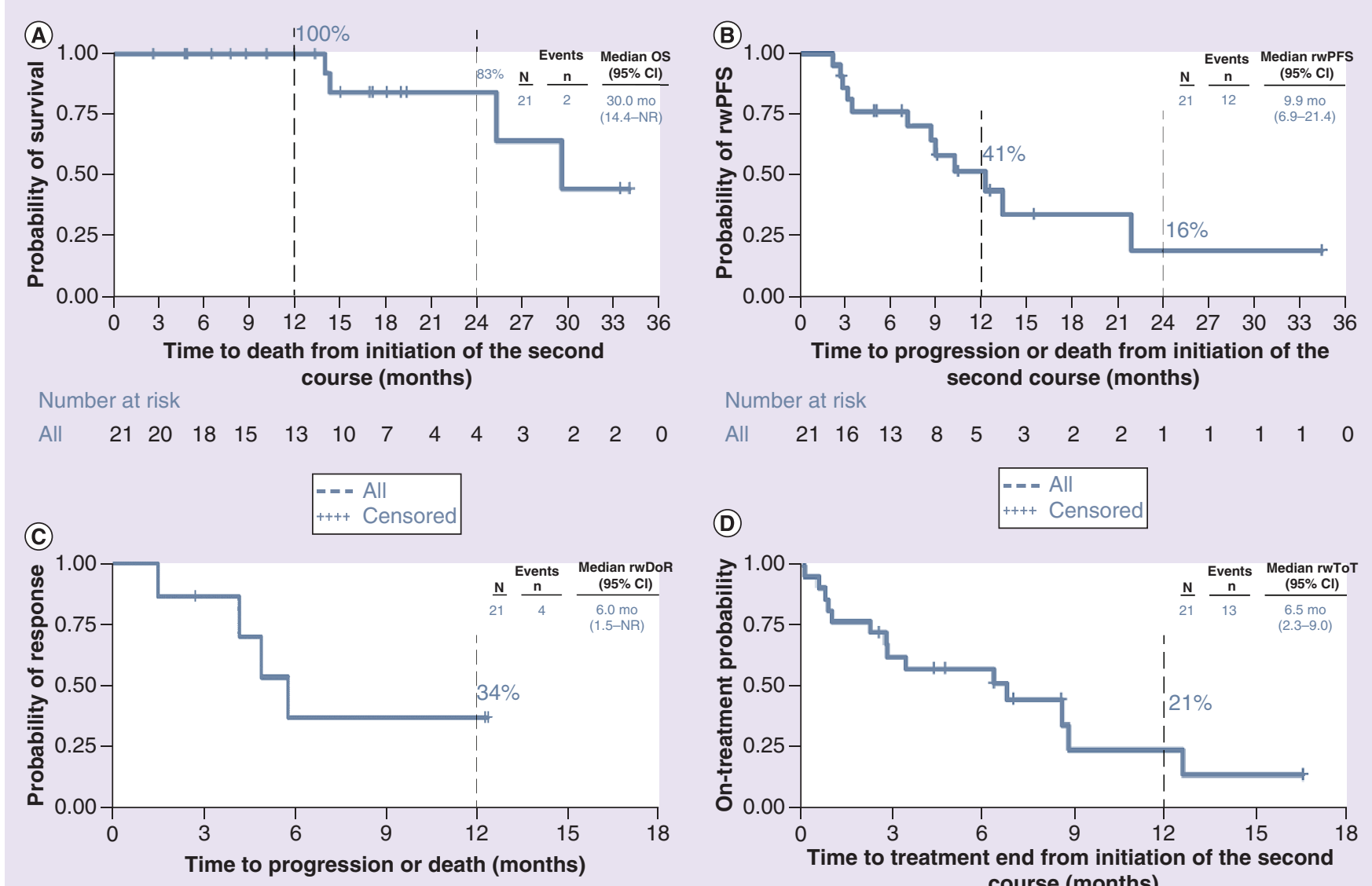

Number at risk
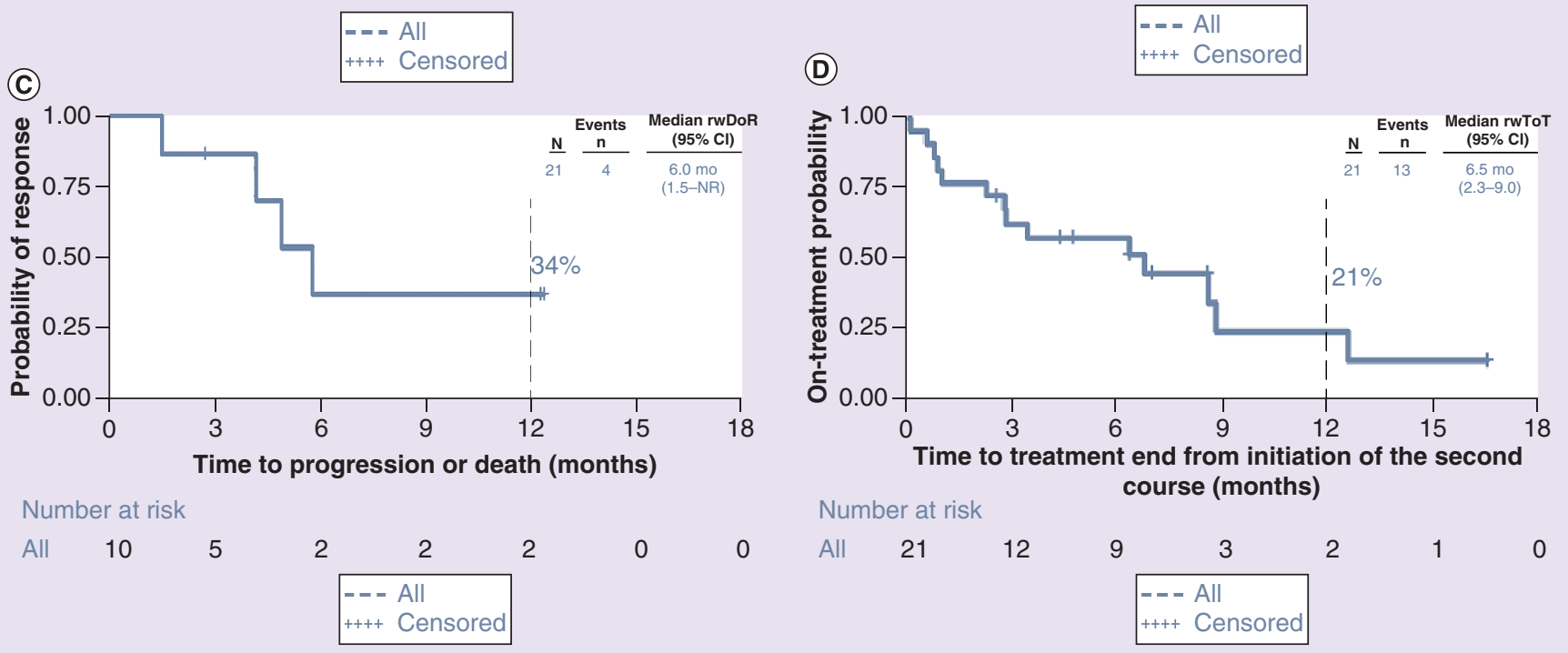

Figure 2. Kaplan-Meier estimates of overall survival, real-world progression-free survival, real-world duration of response and real-world time on treatment. (A) OS, (B) rwPFS, (C) rwDoR, (D) rwToT.

NR: Not reached. OS: Overall survival; rwDoR: Real-world duration of response; rwPFS: Real-world progression-free survival; rwTOT: Real-world time on treatment.

Most prior studies, including clinical trials and cohort studies, have examined the results of retreatment with anti-PD-1 monotherapy for small patient groups. In the KEYNOTE-006 clinical trial, after median follow-up of 14.3 months, among 13 patients treated with a second course of pembrolizumab, three and four achieved CR and PR, respectively (a response rate of 54\%), while three had SD, one PD and two pending response at time of publication [5]. Importantly, while six patients had treatment-related adverse events, none experienced a grade 3 or 4 adverse event, and second-course pembrolizumab was judged to be of acceptable safety. In another clinical trial (KEYNOTE-001), four patients who achieved CR received second-course pembrolizumab with resultant CR (1), $\mathrm{SD}(1)$ and $\mathrm{PD}(2)$ [21].

A cohort study reported by Jansen et al. [8] that followed 185 patients with advanced melanoma treated at academic centers in Europe and Australia included 19 patients who received a second course of anti-PD-1 monotherapy. Of these 19 patients, six (32\%) achieved a new antitumor response including two with CR and four with PR; five experienced SD. All patients in that study had electively discontinued their first course of anti-PD-1 therapy in the absence of disease progression or toxicity. By contrast, in our study, seven patients (35\%) had discontinued first-course anti-PD-1 therapy because of an adverse effect of therapy (the reason for their treatment gap), and five patients (24\%) received corticosteroids $<3$ months before anti-PD-1 reinitiation, hence during the treatment gap, possibly because of an immune-related adverse event in the first course. Results of recent studies indicate an 


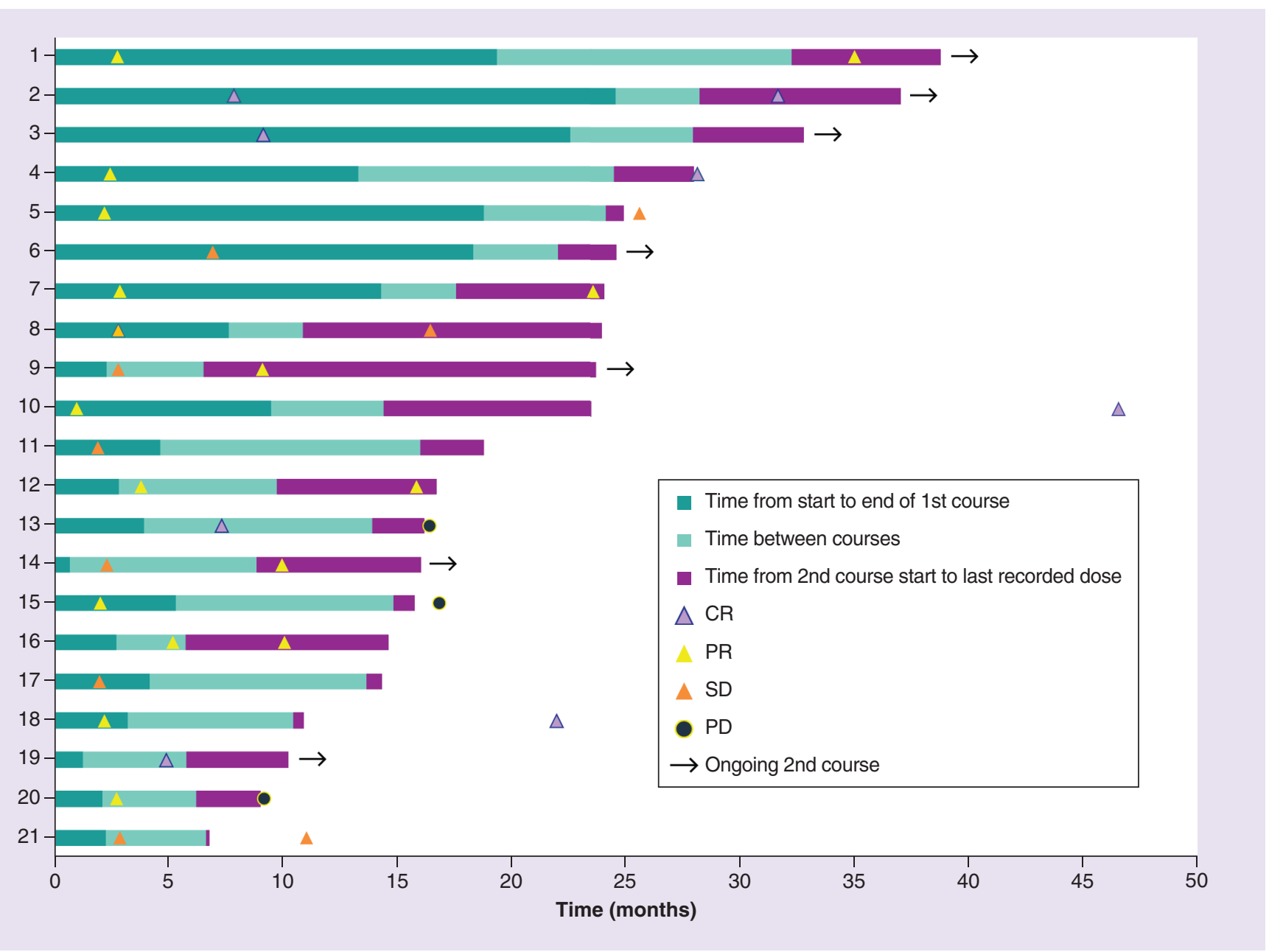

Figure 3. Swimmer plot depicting real-world tumor response and anti-PD-1 monotherapy treatment course for each patient $(n=21)$. Patient \#21 received only one dose of anti-PD-1 monotherapy for the second course.

CR: Complete response; PD: Progressive disease; PR: Partial response; SD: Stable disease.

association of immune-related adverse events with better outcomes (OS and PFS) for patients prescribed anti-PD-1 therapy [22,23], which could at least partially explain the higher response rate in our study than in the study of Jansen et al. [8]. But the numbers are small in both studies and should be interpreted with caution.

More recently, Betof Warner et al. [24] reported on outcomes at a single US hospital for 396 patients with advanced melanoma who received anti-PD-1 monotherapy, including 34 patients who received a second course of anti-PD-1 monotherapy, only five of whom (15\%) responded to retreatment, including two with CR. However, direct comparisons with our study are difficult because of differences in study design and patient eligibility criteria. Eight patients had best response of PD with first-course anti-PD-1, while we required response of SD or better. Moreover, the median duration of retreatment (1.6 months) was much shorter than in our study (median rwToT of 6.5 months), and over half of their patients had surgery or received an interim systemic treatment before the second course of anti-PD-1 monotherapy. In addition, our study excluded patients with mucosal melanoma [24].

Other reports of retreatment with anti-PD-1 monotherapy for metastatic melanoma, all retrospective, have included only three to eight patients, with results that are difficult to interpret because of small patient numbers and/or because other treatment modalities were also employed [9,25,26]. Published case reports of single patients with advanced melanoma who were retreated include varied responses, with some patients achieving PR [27-29], one CR [30] and one with disease progression [31]. Recently reported results of a cohort study including 70 patients with different types of advanced solid tumors (melanoma not explicitly presented) who received retreatment with 
Table 4. Treatment history.

\begin{tabular}{|c|c|}
\hline Variable & $\mathbf{n}=\mathbf{2 1}$ \\
\hline \multicolumn{2}{|l|}{ Treatment line preceding first anti-PD-1 monotherapy course } \\
\hline No prior systemic treatment & $15(71)$ \\
\hline \multicolumn{2}{|l|}{ One prior treatment line: } \\
\hline - Ipilimumab & $4(19)^{\dagger}$ \\
\hline - Cobimetinib-vemurafenib & $1(5)^{\ddagger}$ \\
\hline \multicolumn{2}{|l|}{ Two prior treatment lines: } \\
\hline - Vemurafenib then ipilimumab & $1(5)^{\ddagger}$ \\
\hline Duration of 1st anti-PD-1 course, median (range), months & $4.6(0.7-24.4)$ \\
\hline Length of treatment gap, median (range), months & $5.3(3.0-12.8)$ \\
\hline \multicolumn{2}{|c|}{ Reason for treatment gap between 1st and 2nd anti-PD-1 $(n=20) \S$} \\
\hline Adverse effect of therapy & $7(35)^{\S}$ \\
\hline Other noncancer-related issue(s) & $5(25)$ \\
\hline Drug holiday & $3(15)$ \\
\hline Cancer-related symptom(s) not related to therapy & $2(10)$ \\
\hline Patient request & $2(10)$ \\
\hline Other & $1(5)$ \\
\hline \multicolumn{2}{|l|}{ Reason for discontinuing second-course anti-PD-1 } \\
\hline Adverse effect of therapy & $5(24)$ \\
\hline Disease progression & $3(14)$ \\
\hline Cancer-related symptom(s) not related to therapy & $3(14)$ \\
\hline \multicolumn{2}{|l|}{ Number of treatment lines after second-course anti-PD-1 } \\
\hline 1 line & $7(33)$ \\
\hline 2 lines & $1(5)$ \\
\hline 3 lines & $1(5)$ \\
\hline \multicolumn{2}{|c|}{$\begin{array}{l}\text { Data are } \mathrm{n}(\%) \text {. Percentages may not add up to } 100 \% \text { because of rounding. } \\
\text { †One of these four patients had BRAF-mutant melanoma. } \\
\text { ‡Each of these two patients had BRAF-mutant melanoma. } \\
\S \text { The reason for the treatment gap was missing for one patient, and the percentages apply to the remaining } 20 \text { patients. 'Other' denoted a reason that did not fall into the above } \\
\text { categories. } \\
\text { mo: Month. }\end{array}$} \\
\hline
\end{tabular}

durvalumab after response to the first course of durvalumab suggest restored antitumor efficacy with high rates of disease control [32].

The survival outcomes in our study compare favorably with OS reported in other community-based, retrospective studies in which pembrolizumab was administered for advanced melanoma after other regimens in earlier treatment lines. In another study also utilizing the Flatiron Health database, median OS was 13.9 months (95\% CI: 10.722.7 months) and 12.5 months (8.2-24.8 months) for pembrolizumab administered in second-line $(\mathrm{n}=152)$ and third-line or later $(\mathrm{n}=65)$, respectively [33]. In the study of Cowey et al. [34], median OS of 168 patients across all lines (first-line through third/later-line) was 19.4 months (95\% CI: 14.0-not reached), and 1-year survival was $64 \%$ for pembrolizumab administered in second-line $(n=87)$ and $49 \%$ for pembrolizumab administered in third-line or later $(\mathrm{n}=42)$. However, we note that our study population includes only responders to anti-PD-1 (i.e., to the first course); therefore, the other study populations are not directly comparable because they included all patients who received anti-PD-1 therapy.

Limitations of this study include the small size of the study cohort and, as in all retrospective studies, the potential for selection bias and misclassification errors. Because EHR data were recorded for clinical, not research, purposes, there could also be errors in recording and other shortcomings in quality [12]. The database lacked a complete characterization of all patients' treatment history: the reasons for discontinuation of the first anti-PD-1 course and the reasons for initiating retreatment were not available. However, we do know the reasons for the treatment gap in the majority of patients, and we can assume that anti-PD-1 therapy was reinitiated for clinically valid reasons in the real-world setting of this study. 
We elected to pool the findings, despite the fact that anti-PD-1 therapy could have been stopped and then reinitiated for varying reasons for different patients. Several key characteristics were missing for some patients, most notably ECOG performance status for 33\% of patients and LDH level for 14\%. Both rwP and rwTR, captured retrospectively, served as surrogates for prospectively gathered progression events and tumor response and cannot be considered as reliable as PFS and tumor response assessed by blinded review using RECIST (response evaluation criteria in solid tumors).

This EHR-derived data collected from routine clinical care, despite its limitations, serves to illustrate the outcomes of melanoma therapy in the broad, heterogeneous patient population seen by oncologists outside the more tightly controlled populations and environment of clinical trials. The Flatiron Health database is a large, well-maintained and curated database that is used frequently for observational research $[12,13,33]$. Strengths of this study include the median patient follow-up of longer than 1 year (median, 14.4 months), the stringent identification of retreatment (gap $\geq 90$ days) and the fact that most patients $(86 \%)$ received retreatment at community oncology clinics, where most patients with advanced melanoma are treated in the USA.

Further work is needed to answer several key questions regarding anti-PD-1 monotherapy: when to stop treatment that is well-tolerated? What drug/drug class to use in second-line therapy? How long to retreat? And what patient characteristics or biomarkers might help to clarify the answers to questions one to three $[35,36]$ ?

\section{Conclusion}

Retreatment with anti-PD-1 monotherapy may provide additional benefit for patients with advanced melanoma that had responded to a first course of anti-PD-1 monotherapy with CR, PR or SD. Patients in this retrospective, 'real-world' database study comprising mostly community oncology practices who received a second course of anti-PD-1 monotherapy experienced a high response rate ( $81 \%$ disease control rate), $100 \%$ survival at 1 year, and a median OS of 30.0 months. Longer follow-up and larger, prospective clinical trials are needed to confirm the findings of this study and to better characterize the outcomes of second-course anti-PD-1 monotherapy for advanced melanoma.

\section{Summary points}

- Limited clinical trial data suggest that patients with melanoma who have stable disease (SD) or better after first-course anti-PD-1 monotherapy may benefit from retreatment.

- Our aim was to explore patient characteristics, treatment patterns and outcomes among patients with advanced melanoma at US oncology practices who were retreated with second-course anti-PD-1 monotherapy after having responded or had controlled disease after a first course of anti-PD-1 monotherapy.

- For this retrospective observational study using deidentified electronic health records, eligible patients were adults with unresectable melanoma who received first-course anti-PD-1 monotherapy (nivolumab or pembrolizumab) on/after 4 September 2014, plus retreatment with anti-PD-1 monotherapy after a $\geq 90$-day gap, excluding those with first-course progressive disease (PD).

- Overall survival (OS) was calculated by Kaplan-Meier, and real-world tumor response (rwTR) was determined by enhanced manual chart review and categorized as complete response, partial response, SD or PD.

- Of 21 eligible patients, 12 (57\%) were male; median age was 74 years (range 34-84). Of those with available data, 9/20 (45\%) had BRAF-mutant melanoma, 1/18 (6\%) had elevated LDH level and 4/14 (29\%) had ECOG performance status of 2 .

- At data cutoff (31 October 2018), median follow-up from retreatment initiation was 14.4 months (range, 2.6-34.5 months).

- The median OS was 30.0 months (95\% Cl: 14.4 not reached); 1 -year survival was $100 \%(95 \% \mathrm{Cl}: 100-100 \%)$; and 2-year survival was $83 \%$ (95\% Cl: 48-96\%).

- Response to retreatment (complete response+partial response) was recorded for 10/16 patients (63\%) with known best rwTR; three patients achieved SD and three had PD, while five patients had no recorded rwTR.

- In this study, patients with advanced melanoma who had SD or better after a first anti-PD-1 course benefited from retreatment with anti-PD-1 monotherapy, showing high response rate and $100 \%$ survival at 1 year, with median OS of 30 months.

- Limitations of this study include the small sample size and the heterogeneous study cohort, for which we pooled the findings, despite the fact that anti-PD-1 therapy could have been stopped and then reinitiated for varying reasons for different patients. 


\section{Author contributions}

Conception and design of the study: ED Whitman, W Ou and C Krepler. Data analysis: W Ou. Interpretation of findings, critical review and revision of the manuscript: ED Whitman, E Scherrer, W Ou and C Krepler. All authors read and approved the final manuscript and agree to be accountable for all aspects of the work.

\section{Acknowledgments}

The authors gratefully acknowledge the help of S Tanniru (Merck \& Co., Inc., NJ, USA) for providing study management support and T Shi and W Meng (Merck \& Co., Inc., NJ, USA) for providing statistical programming support.

Financial \& competing interests disclosure

This work was supported by Merck Sharp \& Dohme Corp., a subsidiary of Merck \& Co., Inc., Kenilworth, NJ, USA. The funder of the study participated in development of the study design and funded the analysis of the data. All authors, including those employed by Merck, participated in the data interpretation and writing of the manuscript. ED Whitman is a consultant and serves on advisory boards and/or speaker's bureaus for Merck, BMS, Genentech, Novartis, Amgen, Castle Biosciences, Regeneron and Sanofi Genzyme. E Scherrer, W Ou and C Krepler are full-time employees of Merck Sharp \& Dohme, Corp., a subsidiary of Merck \& Co., Inc., NJ, USA and own stock of Merck \& Co., Inc., NJ, USA. The authors have no other relevant affiliations or financial involvement with any organization or entity with a financial interest in or financial conflict with the subject matter or materials discussed in the manuscript apart from those disclosed.

Medical writing and editorial assistance was provided by EV Hillyer, DVM (freelance). This assistance was funded by Merck Sharp \& Dohme Corp., a subsidiary of Merck \& Co., Inc., NJ, USA.

\section{Ethical conduct of research}

The deidentified dataset supporting the conclusions of this article was provided by Flatiron Health. Ethical approval was obtained before study initiation from the Copernicus Group Institutional Review Board, including a waiver of informed consent for working with deidentified data. The data remained deidentified throughout the analyses to protect patient confidentiality.

\section{Open access}

This work is licensed under the Attribution-NonCommercial-NoDerivatives 4.0 Unported License. To view a copy of this license, visit http://creativecommons.org/licenses/by-nc-nd/4.0/

\section{References}

Papers of special note have been highlighted as: $\bullet$ of interest; $\bullet \bullet$ of considerable interest

1. Gong J, Chehrazi-Raffle A, Reddi S, Salgia R. Development of PD-1 and PD-L1 inhibitors as a form of cancer immunotherapy: a comprehensive review of registration trials and future considerations. J. Immunother. Cancer 6(1), 8 (2018).

2. Eggermont AM, Spatz A, Robert C. Cutaneous melanoma. Lancet 383(9919), 816-827 (2014).

3. Leiter U, Buettner PG, Eigentler TK et al. Hazard rates for recurrent and secondary cutaneous melanoma: an analysis of 33,384 patients in the German Central Malignant Melanoma Registry. J. Am. Acad. Dermatol. 66(1), 37-45 (2012).

4. Robert C, Ribas A, Hamid O et al. Durable complete response after discontinuation of pembrolizumab in patients with metastatic melanoma. J. Clin. Oncol. 36(17), 1668-1674 (2018).

5. Robert C, Ribas A, Schachter J et al. Pembrolizumab versus ipilimumab in advanced melanoma (KEYNOTE-006): post-hoc 5-year results from an open-label, multicentre, randomised, controlled, Phase III study. Lancet Oncol. 20(9), 1239-1251 (2019).

-. Report of 5 years' follow-up in the KEYNOTE-006 study, including outcomes for 13 patients treated with a second course of pembrolizumab.

6. Topalian SL, Sznol M, McDermott DF et al. Survival, durable tumor remission, and long-term safety in patients with advanced melanoma receiving nivolumab. J. Clin. Oncol. 32(10), 1020-1030 (2014).

7. Topalian SL, Hodi FS, Brahmer JR et al. Five-year survival and correlates among patients with advanced melanoma, renal cell carcinoma, or non-small cell lung cancer treated with nivolumab. JAMA Oncol. 5(10), 1411-1420 (2019).

- Report of 5 years' follow-up in a large Phase I clinical trial that included 107 patients with melanoma treated with nivolumab.

8. Jansen YJL, Rozeman EA, Mason R et al. Discontinuation of anti-PD-1 antibody therapy in the absence of disease progression or treatment limiting toxicity: clinical outcomes in advanced melanoma. Ann. Oncol. 30(7), 1154-1161 (2019).

- Large observational study that included findings for 19 patients who were retreated with anti-programmed death 1 monotherapy.

9. Ladwa R, Atkinson V. The cessation of anti-PD-1 antibodies of complete responders in metastatic melanoma. Melanoma Res. 27(2), $168-170$ (2017). 
10. NCCN Clinical Practice Guidelines in Oncology: Melanoma Version 1.2020. (2020). www.nccn.org/professionals/physician_gls/default.aspx

11. Flatiron Health database. (2020). https://flatiron.com/real-world-evidence/

12. Berger ML, Curtis MD, Smith G, Harnett J, Abernethy AP. Opportunities and challenges in leveraging electronic health record data in oncology. Future Oncol. 12(10), 1261-1274 (2016).

- Review of the strengths and limitations of electronic health records as a data source for studies in oncology.

13. O'Connor JM, Fessele KL, Steiner J et al. Speed of adoption of immune checkpoint inhibitors of programmed cell death 1 protein and comparison of patient ages in clinical practice vs pivotal clinical trials. JAMA Oncol. 4(8), e180798 (2018).

14. Curtis MD, Griffith SD, Tucker M et al. Development and validation of a high-quality composite real-world mortality endpoint. Health Serv. Res. 53(6), 4460-4476 (2018).

15. Carrigan G, Whipple S, Taylor MD et al. An evaluation of the impact of missing deaths on overall survival analyses of advanced non-small cell lung cancer patients conducted in an electronic health records database. Pharmacoepidemiol. Drug Saf. 28(5), 572-581 (2019).

16. Velcheti V, Chandwani S, Chen X, Pietanza MC, Burke T. First-line pembrolizumab monotherapy for metastatic PD-L1-positive NSCLC: real-world analysis of time on treatment. Immunotherapy. 11(10), 889-901 (2019).

17. Friends of Cancer Research (2018). Establishing a framework to evaluate real-world endpoints. (2020). www.focr.org/publications/establishing-f ramework-evaluate-real-world-endpoints

18. Velcheti V, Chandwani S, Chen X, Pietanza MC, Piperdi B, Burke T. Outcomes of first-line pembrolizumab monotherapy for PD-L1-positive (TPS > /=50\%) metastatic NSCLC at US oncology practices. Immunotherapy 11(18), 1541-1554 (2019).

19. Griffith SD, Tucker M, Bowser B et al. Generating real-world tumor burden endpoints from electronic health record data: comparison of RECIST, radiology-anchored, and clinician-anchored approaches for abstracting real-world progression in non-small cell lung cancer. Adv. Ther. 36(8), 2122-2136 (2019).

20. Griffith SD, Miksad RA, Calkins G et al. Characterizing the feasibility and performance of real-world tumor progression end points and their association with overall survival in a large advanced non-small-cell lung cancer data set. JCO Clin. Cancer Inform. 3, 1-13 (2019).

- Study exploring the use of real-world cancer progression endpoints derived from deidentified electronic health record data.

21. Hamid O, Robert C, Daud A et al. Five-year survival outcomes for patients with advanced melanoma treated with pembrolizumab in KEYNOTE-001. Ann. Oncol. 30(4), 582-588 (2019).

22. Das S, Johnson DB. Immune-related adverse events and anti-tumor efficacy of immune checkpoint inhibitors. J. Immunother. Cancer 7(1), 306 (2019).

23. Indini A, Di Guardo L, Cimminiello $\mathrm{C}$ et al. Immune-related adverse events correlate with improved survival in patients undergoing anti-PD1 immunotherapy for metastatic melanoma. J. Cancer Res. Clin. Oncol. 145(2), 511-521 (2019).

24. Betof Warner A, Palmer JS, Shoushtari AN et al. Long-term outcomes and responses to retreatment in patients with melanoma treated with PD-1 blockade. J. Clin. Oncol. 38(15), 1655-1663 (2020).

- Large observational study with long-term follow-up of 396 patients with unresectable melanoma after discontinuation of anti-programmed death 1 therapy, including outcomes for 34 patients retreated with anti-PD1 monotherapy.

25. Blasig H, Bender C, Hassel JC et al. Reinduction of PD1-inhibitor therapy: first experience in eight patients with metastatic melanoma. Melanoma Res. 27(4), 321-325 (2017).

26. Nomura M, Otsuka A, Kondo $\mathrm{T}$ et al. Efficacy and safety of retreatment with nivolumab in metastatic melanoma patients previously treated with nivolumab. Cancer Chemother. Pharmacol. 80(5), 999-1004 (2017).

27. Lipson EJ, Sharfman WH, Drake CG et al. Durable cancer regression off-treatment and effective reinduction therapy with an anti-PD-1 antibody. Clin. Cancer Res. 19(2), 462-468 (2013).

28. Bernard-Tessier A, Baldini C, Martin P et al. Outcomes of long-term responders to anti-programmed death 1 and anti-programmed death ligand 1 when being rechallenged with the same anti-programmed death 1 and anti-programmed death ligand 1 at progression. Eur. J. Cancer 101, 160-164 (2018).

29. Lepir T, Zaghouani M, Roche SP et al. Nivolumab to pembrolizumab switch induced a durable melanoma response: a case report. Medicine (Baltimore) 98(2), e13804 (2019).

30. Kilickap S, Guven DC, Aktepe OH, Aktas BY, Dizdar O. Complete responses to two different anti-PD1 agents in a metastatic melanoma patient. J. Oncol. Pharm. Pract. 26(2), 496-499 (2019).

31. Martini DJ, Lalani AA, Bosse D et al. Response to single agent PD-1 inhibitor after progression on previous PD-1/PD-L1 inhibitors: a case series. J. Immunother. Cancer 5(1), 66 (2017).

32. Sheth S, Gao C, Mueller N, Martinez P, Soria J-C. Durvalumab activity in previously treated patients who stopped durvalumab without disease progression (abstract 1175O). Ann. Oncol. 30(Suppl. 5), v475-v476 (2019).

33. Liu FX, Ou W, Diede SJ, Whitman ED. Real-world experience with pembrolizumab in patients with advanced melanoma: a large retrospective observational study. Medicine (Baltimore) 98(30), e16542 (2019). 
34. Cowey CL, Liu FX, Black-Shinn J et al. Pembrolizumab utilization and outcomes for advanced melanoma in US community oncology practices. J. Immunother. 41(2), 86-95 (2018).

35. Beaver JA, Hazarika M, Mulkey F et al. Patients with melanoma treated with an anti-PD-1 antibody beyond RECIST progression: a US Food and Drug Administration pooled analysis. Lancet Oncol. 19(2), 229-239 (2018).

36. Gauci ML, Lanoy E, Champiat $S$ et al. Long-term survival in patients responding to anti-PD-1/PD-L1 therapy and disease outcome upon treatment discontinuation. Clin. Cancer Res. 25(3), 946-956 (2019). 
